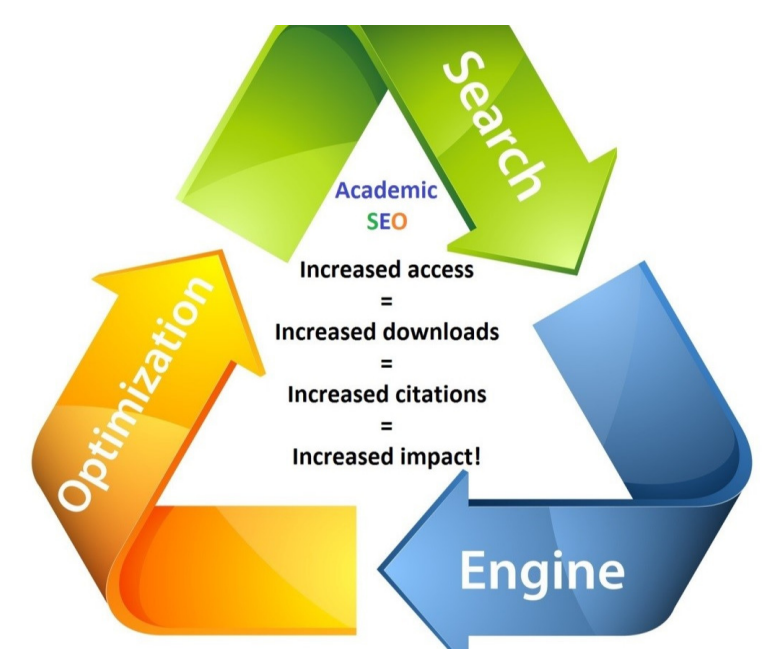

\title{
Optimize Your Article for Search Engine
}

\author{
Nader Ale Ebrahim \\ 1 Universiti Malaya
}

\section{Abstract}

This article provides guidelines on how to optimize scholarly literature for academic search engines like Google Scholar, in order to increase the article visibility and citations.

\section{Introduction}

Academic search engine optimization (ASEO) is a debatable topic [1]. Some researchers believe “Designing scientific articles just for SEO would undermine the credibility and definitively the quality of the articles" [2]. On the other hand, the inclusion of the articles in the index improves the ability to make their articles available to the academic community [3]. "If you want your paper be "accepted" by a search eng ine you have to accept the search eng ines style guidelines" [2]. The literature indicates a significant and positive relationship between both citation in Google Scholar and ISI Web of Science with the number of versions. Increasing the visibility means raises the number of versions of an article and vice versa. The number of "versions" will be accessible in a Google Scholar search result.

\section{Discussion}

There are several techniques that you can optimize your article for search eng ines [1, 4]: 
1. Usage of strong keywords and synonyms; Once the keywords are chosen, they need to be mentioned in the right places: in the title, and as often as possible in the abstract and the body of the text (but, of course, not so often as to annoy readers);

2. The completeness of documents metadata; When documents are converted to PDF, all metadata should be correct (especially author and title). Some search engines use PDF metadata to identify the file or to display information about the article on the search results page. It may also be beneficial to give a meaning ful file name to each article;

3. Write a good and short title;

4. Consistent spelling of authors' first and last names;

5. Refer to reliable resources with high citations;

6. Select a proper journal; and

7. Deposit your paper in different repository.

Google Scholar as one of the academic search eng ine indexes scholarly material from proprietary sources such as subscription and open access journals, conference proceedings and white papers. Google Scholar weighted heavily the title, the Journal name and author names also multiple versions of an article, (e.g. on your home page and in UM Research Repository) [1].

Beel, Gipp and Wilde [1] believe that academic search eng ine optimization (ASEO) should be a common procedure for researchers, similar to, selecting an appropriate journal for publication. ASEO should not be seen as a guide on how to cheat academic search engines. Rather, it is about helping academic search eng ines to understand the content of research papers and, thus, about how making this content more widely and easily available.

\section{References}

[1] J. Beel, et al., "Academic Search Eng ine Optimization (ASEO)," Journal of Scholarly Publishing, vol. 41, no. 2, pp. 176-190, 01/01/, 2010.

[2] http://blog.webometrics.org.uk. "Academic Search Engine Optimization: An inevitable evil?,".

[3] J. Beel, and B. Gipp, "On the robustness of google scholar against spam," in Proceedings of the 21st ACM conference on Hypertext and hypermedia $\left(H T^{\prime} 10\right)$, Toronto, Ontario, Canada, 2010, pp. 297-298.

[4] N. Ale Ebrahim, et al., "Effective Strategies for Increasing Citation Frequency," International Education Studies, vol. 6, no. 11, pp. 93-99, October 23, 2013. 
